10 Prytherch D, Sirl J, Schmidt P et al. The use of routine laboratory data to predict inhospital death in medical admissions. Resuscitation 2005;66:203-7.

11 Gao H, McDonnell A, Harrison DA et al. Systematic review and evaluation of physiological track and trigger warning systems for identifying at-risk patients on the ward. Intensive Care Med 2007;33:667-79.

12 Royal College of Physicians. Acute medical care. The right person, in the right setting first time. Report of the Acute Medicine Task Force. London: RCP, 2007.

13 Trzeciak S, Chansky ME, Dellinger RP et al. Operationalizing the use of serum lactate measurement for identifying high risk of death in a clinical practice algorithm for suspected severe sepsis. Acad Emerg Med 2006;13(Suppl 1):S150-b-1.

14 Phua J, Koay ES, Lee KH. Lactate, procalcitonin, and amino-terminal pro-B-type natriuretic peptide versus cytokine measurements and clinical severity scores for prognostication in septic shock. Shock 2008;29:328-33.

15 Levy MM, Dellinger RP, Townsend SR et al. The Surviving Sepsis Campaign: results of an international guideline-based performance improvement program targeting severe sepsis. Review. Intensive Care Med 2010;36:222-31.

16 Bochud PY, Bonten M, Marchetti O, Calandra T. Antimicrobial therapy for patients with severe sepsis and septic shock: an evidence-based review. Crit Care Med 2004;32(11 Suppl):S495-512.

17 Rivers E, Nguyen B, Havstad S et al. Early goal-directed therapy in the treatment of severe sepsis and septic shock. $N$ Engl J Med 2001;345:1368-77.

18 Ventilation with lower tidal volumes as compared with traditional tidal volumes for acute lung injury and the acute respiratory distress syndrome. The Acute Respiratory Distress Syndrome Network. $N$ Engl J Med 2000;342:1301-8.

19 Angus DC. The search for effective therapy for sepsis: back to the drawing board? JAMA 2011;306:2614-5.

Address for correspondence: Dr J Soong, RCP Clinical Quality Improvement Research Fellow NIHR CLAHRC for Northwest London team, 4th Floor, Lift Bank D, Imperial College London Chelsea \& Westminster Hospital NHS Foundation Trust, 369 Fulham Road, London SW10 9NH. Email: j.soong@imperial.ac.uk

\title{
Poisoning and self-harm
}

\section{N Bateman, professor in clinical pharmacology, National Poisons Information Service, Edinburgh}

This article will deal with several areas in which there have been recent problems or developments in knowledge and practice in relation to poisoning and self-harm.

\section{Initial triage, consent and management}

A confidential deaths enquiry conducted on patients who died in English hospitals in 2005 from poisons-related events suggested that the major initial errors in care were delay in appropriate rapid assessment and airway management. ${ }^{1}$ A reduced conscious level, in particular a Glasgow Coma Scale below 8 , is a recognised risk factor for increased mortality in poisonings with central nervous system (CNS) effects. Rapid changes in conscious level early in admission are a recognised additional risk factor. Therefore careful monitoring and management of these patients in the initial phases are essential.

\section{Do not resuscitate}

The study also revealed that a number of patients presented to hospital with documents indicating they did not wish to be resuscitated. ${ }^{1}$ Such documents may have legal authority, but it is important to be clear about the particular scenarios they cover and whether they do have legal authority. Use of such a document in the case of a young woman with repeated selfharm behaviour who subsequently died of ethylene glycol poisoning stimulated an academic exchange in the British Medical Journal emphasising the care needed in assessing this type of situation. Decisions made by patients under stress with psychological disorders may not be as clear-cut as they initially appear. Careful psychosocial assessment is an essential cornerstone in the management of self-harm patients. ${ }^{2,3}$

\section{Paracetamol}

Paracetamol remains the most common cause of overdose in the UK, yet management errors continue to occur. A recent study demonstrated the benefits of using a multidisciplinary care plan in this frequent clinical scenario. ${ }^{4}$ Benefits of this approach include better processes of care, with appropriate use of the antidote $\mathrm{N}$-acetyl cysteine. Copies of this care plan are available for UK health professionals to download from TOXBASE; ${ }^{5}$ this provides a comprehensive guide to assessment and management of poisoning.

\section{Drugs of abuse}

The most rapidly changing area of clinical toxicology has been the increase in range and toxicity of so-called 'legal highs' (Table 1). As illicit drug regulation changes to take an account of new molecular structures, so new chemicals are marketed to young people to try to get round the legislation. From a clinician's perspective, this creates a particular problem since it occurs increasingly frequently and the names on the products bear little resemblance to their actual contents. Indeed, the same name may be used for different ingredients over a time period as drug regulation changes what is legally permissible to sell.

An example is the 'ivory wave' product that changed drug content as legislation banned its original constituents. It initially contained desoxypipradol, a drug previously appearing in Ireland under the name 'whack'. It had been investigated as a potential antidepressant because of its stimulant effects and was subsequently marketed in the UK as 'ivory wave'. It produced significant physical and psychological symptoms, particularly formication (tactile sensation of being crawled over by insects) and was also associated with rebound self-harm and suicide. ${ }^{6}$ Early identification of this product in 'ivory wave' and its potential adverse effects rapidly led to commercial suppliers changing its psychoactive ingredients, while maintaining its 'trade name' on the internet where it continues to be sold.

Other recent examples include the production of more potent cannabinoid compounds which appear to have more psychoactive action than traditional 
cannabinoids ('herbal spice'), and the use of pharmaceutical products in 'herbal' pills. The latter products may appear in different countries under different names. ${ }^{7}$

There seem to be at least 150 different chemicals with cannabinoid properties. In overdose they may cause symptoms of extreme cannabis use, but seizures and hypokalaemia are also reported. They appear to have greater affinity for the CB1 cannabis receptor than traditional products which contain delta-9-tetrahydrocannabinol as their active ingredient. ${ }^{8}$

A range of drugs of abuse is available, in general acting as CNS stimulants causing effects similar to amfetamine (Table 1). Key differences appear to be in their duration of action, propensity to cause psychosis, hyperpyrexia, rhabdomyolysis and cardiovascular features. As with desoxypipradol, late after effects may include depressive reaction or continuing psychosis. ${ }^{6}$ Patients who ingest these compounds therefore need to be monitored over a period of hours to assess their full impact.

\section{Management of cardiotoxicity}

\section{Drugs cardiotoxic in overdose}

Many routinely prescribed drugs cause cardiotoxicity in overdose. The most frequent causes of severe heart failure in poisoning include:

- calcium channel blockers (CCB)

- beta-blockers

- sodium channel blockers

- local anaesthetic agents

- tricyclic antidepressants

- drugs of abuse including cocaine.

Cardiotoxicity, like CNS depression, is therefore a major concern in accidental poisoning and overdose. Two new treatment strategies, insulin/glucose (dextrose) and intralipid, have emerged over the past five years with the potential to improve outcome in cases of cardiotoxicity.

In addition, improved technologies such as extracorporeal assist devices make cardiac support more readily available. These may have a role in the most severe poisoning where other treatments have failed to ensure adequate cardiac output and the poison is thought not to have produced irreversible CNS damage.

\section{Table 1. Examples of drugs that have been used in 'legal highs'.}

\section{Drug group}

Class

Amfetamines and cathinones:

- mephedrone

- methcathinone

- methedrone

- methylenedioxypyrovalerone (MDPV)

- naphyrone

Desoxypipradol (2-DPMP)

(not an amfetamine or cathinone by structure but similar toxicity)

Piperazines:

- benzylpiperazine (BZP)

- meta-chlorophenylpiperazine (mCPP)

- methylenedioxybenzylpiperazine (MDBP)

- trifluoromethylphenylpiperazine (TFMPP)
Now Class B substance

All now Class B substance

\author{
(n)
}

Now Class $C$ substance

Now Class $C$ substance

Now Class C substance

\section{Box 1. Indications and treatment regimen for intra lipid.}

Intralipid should be used for drugs with local anaesthetic toxicity at the onset of neurological or cardiovascular symptoms. It should be considered in other haemodynamically significant intoxication from fat soluble drugs (eg propranolol, amitriptyline) after general supportive measures and recognised antidotes have been unsuccessful.

No optimal regimen is established: currently, a $1.5 \mathrm{ml} / \mathrm{kg}$ bolus, followed by $0.25-0.5 \mathrm{ml} / \mathrm{kg} /$ min over 30-60 min is an appropriate starting approach.

The bolus may be repeated in cases of cardiac arrest. Titrating the infusion rate to the clinical response and repeating lipid administration at the onset of recurrent deterioration is reasonable.

\section{Table 2. Metabolic problems in myocardial function in severe poisoning}

- Switch from free fatty acid to carbohydrate energy sources.

- Relative lack of insulin precludes optimal energy production, resulting in impaired cardiac performance.

- Carbohydrate delivery and glycolysis are impaired due to poor tissue perfusion and acidosis.

- Lactic acidosis results not only from tissue hypoperfusion but also from activation of mitochondrial dehydrogenase.

\section{Key points}

Initial assessment and triage of patients with poisoning remains a challenge, particularly with regard to validity and appropriateness of 'advance directives' to this situation

Careful monitoring is required if there is respiratory depression (GCS 8 or less)

Multidisciplinary care pathways improve care of paracetamol poisoning

Stimulant drugs of abuse come in many forms and users are usually unaware of ingredients. Unexpected and delayed toxicity is still seen from new 'legal highs'

Managing severe cardiotoxicity requires critical care input; new management approaches include insulin and intralipid, but their roles in therapy are yet to be clearly defined

Keywords: self harm, poisoning, triage, legal highs, cardiotoxicity, paracetamol 


\section{Intralipid}

Therapy with intralipid was introduced following initial experimental work on the isolated heart and subsequently on animal models, which suggested that drug partitioning into lipid could change the pharmacodynamics of drug action, resulting in clinical improvement. Subsequent use in clinical case series has confirmed this initial impression and lipid therapy has now been used in the management of a wide range of toxins. At present, it is therefore difficult exactly to place its most effective role. Theoretically, patients who have ingested lipid soluble drugs with actions on sodium channels should benefit. ${ }^{9}$ Experimental studies suggest that water-octanol coefficient (ie degree of relative lipid solubility) should predict clinical benefit. Recent in vitro studies also suggest that lipid may have a direct effect on sodium channels, modulating their function. Expert advice should be sought. Dose and suggested indications for use of intralipid are shown in Box 1. 9

\section{Insulin and dextrose}

Insulin and dextrose therapy was first introduced in 1999 based on experimental data suggesting insulin has inotropic properties. Its use has subsequently become more widespread, particularly in the management of CCB poisoning, the severity of which relates to the degree of hyperglycaemia, causing a complex effect on the heart with several metabolic consequences (Table 2). Interestingly, insulin appears to act on all these mechanisms, increasing cardiac efficiency and hence cardiac output.
Hypoglycaemia is a potential risk of using insulin, but can be readily managed in the clinical scenario of CCB poisoning by the additional use of glucose. In a case series of 12 patients, hypoglycaemia was the greatest risk but outcomes were generally good. The only poor outcome in this series resulted from a failure to comply with the agreed management protocol involving insulin. ${ }^{10}$

The exact dose of insulin required can be titrated; doses up to $10 \mathrm{U} / \mathrm{kg} / \mathrm{hr}$ are well described as efficacious and safe.

\section{Conclusions}

The key to the treatment of any patient is careful observation, clinical management and preparedness for response to expected complications. Thirty years ago gastric lavage was the norm, whereas it is now virtually never used in North America, the UK and Western Europe. Changes in management mandate the need for appropriate clinical studies which remains a problem for much of clinical toxicology. Multicentre clinical trials of the new treatments mentioned in this article are required. Until appropriate funding and resource can be identified, it seems reasonable to base changes in treatment on well conducted carefully reported case studies. New drugs of abuse are likely to continue to be developed, and it is important to share experiences with others as these case reports and series arise.

\section{References}

1 Kapur N, Clements C, Bateman N et al. Self-poisoning suicide deaths in England: could improved medical management contribute to suicide prevention? QJM 2010;103:765-75.

2 David AS, Hotopf M, Moran P et al. Mentally disordered or lacking capacity? Lessons for management of serious deliberate self harm. BMJ 2010;341:c4489.

3 Kapur N, Clements C, Bateman N et al. Advance directives and suicidal behaviour. BMJ 2010;341:c4557.

4 Pettie JM, Dow MA, Sandilands EA et al. An integrated care pathway improves the management of paracetamol poisoning. Emerg Med 2011; May 11 (ahead of print).

5 TOXBASE. www.TOXBASE.org

6 Murray DB, Potts S, Haxton C et al. 'Ivory wave' toxicity in recreational drug users; integration of clinical and poisons information services to manage legal high poisoning. Clin Toxicol (Phila) 2012;50:108-13.

7 James D, Adams RD, Spears R et al. Clinical characteristics of mephedrone toxicity reported to the U.K. National Poisons Information Service. Emerg Med J 2011;28:686-9.

8 Atwood BK, Lee D, Straiker A et al. CP47,497-C8 and JWH073, commonly found in 'Spice' herbal blends, are potent and efficacious $\mathrm{CB}(1)$ cannabinoid receptor agonists. Eur J Pharmacol 2011;659:139-45.

9 Jamaty C, Bailey B, Larocque E et al. Lipid emulsions in the treatment of acute poisoning: a systemic review of human and animal studies. Clin Toxicol (Phila) 2010;48:1-27.

10 Holger JS, Stellpflug SJ, Cole JB et al. Highdose insulin: a consecutive case series in toxin-induced cardiogenic shock. Clin Tox (Phila) 2011;49:653-8.

\author{
Address for correspondence: \\ Professor D N Bateman, NPIS \\ Edinburgh, Royal Infirmary of \\ Edinburgh, Edinburgh EH10 4SA. \\ Email: Nick.Bateman@luht.scot.nhs.uk
}

